\title{
How to open a Ryanodine Receptor
}

\author{
Cell Research (2016) 26:1073-1074. doi:10.1038/cr.2016.106; published online 9 September 2016
}

\begin{abstract}
Ryanodine Receptors are large ion channels responsible for the release of $\mathrm{Ca}^{2+}$ from the Endoplasmic and Sarcoplasmic Reticulum, a prerequisite for muscle contraction. Recent cryo-electron microscopy data have allowed a direct visualization of allosteric motions within these membrane protein giants.
\end{abstract}

The human genome encodes three Ryanodine Receptors (RyR) isoforms consisting of $\sim 5000$ amino acid subunits that assemble into $>2.2 \mathrm{MDa}$ homotetramers. RyR1 is the primary skeletal muscle isoform and is the target for hundreds of mutations linked to central core disease and malignant hyperthermia. RyR2 predominates in the heart and is linked to stress-induced cardiac arrhythmias [1].

Due to their massive size, RyRs have been ideal targets for cryo-electron microscopy (cryo-EM) studies. With continued improvements in technology, these have culminated in three reports on RyR 1 in closed states between 6.1 and $3.8 \AA$, allowing up to $70 \%$ of the structure to be built [2-4]. RyRs are clear members of the superfamily of six-transmembrane (6-TM) ion channels, containing a transmembrane region with a pore-forming domain with inner (S5) and outer helices (S6), and a 4-helix bundle (S1-S4) that resembles voltage-sensing domains in voltagegated channels. Unique to RyRs is a massive cytosolic cap that consists for the most part of a large $\alpha$-helical scaffold, interspersed by a number of globular domains (Figure 1).

RyRs represent the pinnacle of complexity in ion channels, as they are targeted by tens of auxiliary proteins and small molecules that can affect their gating properties [1]. What are the allosteric transitions that govern opening and closing? Again, cryo-EM has proven to be invaluable in solving this puzzle. In 2009, Samso et al. [5] compared open and closed RyR1 at $\sim 10$ $\AA$ resolution, and this work has now been further refined at higher resolution by Wei et al. [6] and Bai et al. [7] in Cell Research. Both groups come to similar conclusions on the movements within the transmembrane region, and offer distinct viewpoints about the role of the cytosolic cap.

RyR1 has a bell-shaped dependence on cytosolic $\mathrm{Ca}^{2+}$ for opening, with small increases promoting channel opening, and higher increases causing inhibition [8]. Armed with $50 \mu \mathrm{M} \mathrm{Ca}^{2+}$ and $10 \mu \mathrm{M}$ PCB95, a potent opener, Bai et al. set out to obtain a cryo-EM structure of an open RyR1, but found the pore region to be in a closed state. Changing the detergent from Tween-20 to CHAPS yielded an open RyR1 structure at 5.7 $\AA$ A. Wei et al. adopted a different strategy, with $100 \mu \mathrm{M} \mathrm{Ca}^{2+}$ and Ruthenium Red, a blocker that further stabilizes the open state. Together with the previous open state structures, and an $8.5 \AA$ structure of the inactivated state in $10 \mathrm{mM} \mathrm{Ca}^{2+}$ [4], there are now multiple structures for pair-wise comparisons.

The opening transitions within the pore-forming domain are as expected for members of the 6-TM ion channel family. The inner helix S6 bends around a conserved glycine hinge, resulting in a widening of the pore around a hydrophobic constriction point, allowing ample space for a hydrated $\mathrm{Ca}^{2+}$ to permeate. These motions are coupled directly to the voltage-sensing like domain (VSL), and the extension of S6. The inner helix is much longer than usual in 6-TM channels, and extends towards a Zinc-finger motif that makes intimate interactions with an upstream 'U-motif' (Figure 1). This offers two pathways to open the RyR: movement of the extended S6 helix, coupled to the U-motif, or movement of the VSL, which is also intimately coupled to the pore domain. Wei et al. describe additional details, including a widening of the selectivity filter, and a movement of the S4 segment towards the lumen upon opening [6].

What are the movements outside the immediate vicinity of the TM region? The activating $\mathrm{Ca}^{2+}$ binding site lies elsewhere, thus the described movements in the TM region must be the result of other conformational changes in the cytosolic cap. Here the answer highly depends on the vantage point, because Bai et al. have shown that there are multiple possible conformations in the cytosolic cap for a closed channel [7]. Using the previously reported $3.8 \AA$ structure of closed RyR 1 as a reference [2], Wei et al. describe the movements of the cap as a large breathing motion, with an increase in the width and height of the cytosolic cap (Figure 1). The socalled central domain plays the role of 'relay', linking these movements to the U-motif and thus the TM region. Right around the 4-fold symmetry axis, the region of the cap containing the $\mathrm{N}$ terminal domains moves upward and outward, confirming previous studies [5, 9]. Bai et al. already see similar movements of the cap in distinct closed states. They describe a total of 4 different 


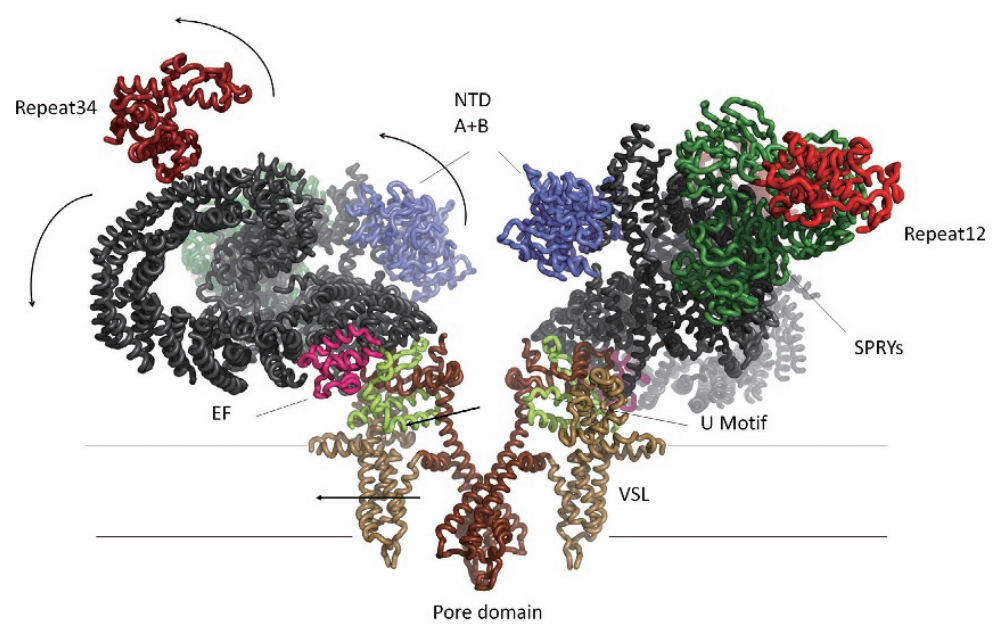

Figure 1 Cartoon model of RyR1 in the open state, as viewed from within the plane of the Sarcoplasmic Reticulum membrane. Only two subunits are shown for clarity. The $\alpha$-helical scaffold is shown in dark grey, with all other domains in colors and labeled. Large arrows indicate the types of motions that happen during opening or within the various closed states of RyR1. VSL: voltage-sensing like domain; NTD A+B: N-terminal domains A and B; EF: EF-hand like domain; SPRYs: SPRY domains 1-3.

closed states (C1-C4), whereby the cap movements are gradually larger going from $\mathrm{C} 1$ to $\mathrm{C} 4$. This is an important finding, because it suggests that the cap can be in an activated position that favors an open channel, but does not command it. The cap of the $\mathrm{C} 3$ state appeared closest to the cap of the open channel, although there are still some differences [7].

Where does the movement originate? Wei et al. point to an EF-hand region as the prime suspect, as it was reported before that mutations in this area have an effect on $\mathrm{Ca}^{2+}$ sensitivity [10]. A more recent study showed that deletion of the entire EF-hand domain did not affect $\mathrm{Ca}^{2+}$-dependent activation of RyR2, suggesting that the EF-hand is not the primary $\mathrm{Ca}^{2+}$ sensor [11]. Despite this, Wei et al. uncover conformational changes within the first EF-hand motif, with a $33^{\circ}$ change in tilt between its helices. $\mathrm{Ca}^{2+}$ could not be observed directly, therefore higher-resolution studies of a $\mathrm{Ca}^{2+}$ bound RyR1 are required to unambiguously find the activating $\mathrm{Ca}^{2+}$-binding site.

These new studies are another leap forward in cracking the RyR puzzle. Next await complexes with the multi- tude of other ligands and proteins that can affect its gating. Of prime interest is the interaction with $\mathrm{Ca}_{\mathrm{v}} 1.1$, the skeletal muscle voltage-gated calcium channel that is known to couple mechanically to RyR1. Voltage-induced changes in the $\mathrm{Ca}_{\mathrm{v}} 1.1$ conformation must somehow trigger an activated state of the RyR1 cytosolic cap. To be continued...

\section{Filip Van Petegem ${ }^{1}$}

${ }^{1}$ University of British Columbia, Department of Biochemistry and Molecular Biology, 2350 Health Sciences Mall, V6T 1 Z3 Vancouver, BC, Canada

Correspondence: Filip Van Petegem

E-mail: filip.vanpetegem@gmail.com

\section{References}

1 Van Petegem F. J Biol Chem 2012; 287:31624-31632.

2 Yan Z, Bai XC, Yan C, et al. Nature 2015; 517:50-55.

3 Zalk R, Clarke OB, des Georges A, et al. Nature 2015; 517:44-49.

4 Efremov RG, Leitner A, Aebersold R, et al. Nature 2015; 517:39-43.

5 Samso M, Feng W, Pessah IN, et al. PLoS Biol 2009; 7:e85.

6 Wei R, Wang X, Zhang Y, et al. Cell Res 2016; 26:977-994.

7 Bai XC, Yan Z, Wu J, et al. Cell Res 2016; 26:995-1006.

8 Meissner G. J Biol Chem 1986; 261:63006306.

9 Kimlicka L, Lau K, Tung CC, et al. Nat Commun 2013; 4:1506.

10 Fessenden JD, Feng W, Pessah IN, et al. J Biol Chem 2004; 279:53028-53035.

11 Guo W, Sun B, Xiao Z, et al. J Biol Chem 2016; 291:2150-2160. 